\title{
Prescrição de caminhada não supervisionada, risco cardiovascular e aptidão física
}

CDD. 20.ed. 616.12

796.07

\author{
Andréia Cristiane Carrenho QUEIROZ* \\ Leandro Campos de BRITO* \\ Mayara Alves dos SANTOS* \\ Rafael Yokoyama FECCHIO* \\ Ana Luiza Bonilha STOCCO* \\ Alan Irwin BEZERRA* \\ Andreza Aguida Pereira CAVALI* \\ Bruno Timóteo MODESTO* \\ Crivaldo Gomes CARDOSO JUNIOR ${ }^{* *}$ \\ Teresa BARTHOLOMEU* \\ Tais TINUCCI* \\ Claudia Lúcia de Moraes FORJAZ*
}

*Escola de Educação

Física e Esporte

Universidade de São

Paulo.

${ }^{* *}$ Centro de Educação

Física e Esporte,

Universidade Estadual

de Londrina.

\section{Resumo}

Objetivo: Avaliar, numa situação real de atuação prática, o efeito da prescrição individualizada de caminhada sem supenvisão da prática sobre o risco cardiovascular e a aptidão física de usuários de um parque público. Métodos: 186 sujeitos (62 \pm 10 anos) foram orientados a caminhar pelo menos $3 x / \mathrm{sem}$, por $30 \mathrm{~min}$, com intensidade de 50 a 80\% da frequência cardiaca de reserva e a fazer alongamentos antes e após a caminhada. A aptidão física e os fatores de risco cardiovascular foram avaliados pré e pós-intervenção. A análise dos dados foi dividida em duas fases: 1) análise na amostra total; 2) análise nos indivíduos com fatores de risco alterados. Os dados foram comparados pelo teste $t$ pareado. Resultados: $\mathrm{Na}$ amostra total, a aptidão física melhorou nos testes de marcha estacionária $(+8,1 \pm 14,5$ passos, $p<0,05)$, impulsão vertical $(+0,5 \pm 2,7 \mathrm{~cm}, p<0,05)$, flexibilidade lombar $(+1,1 \pm 4,7 \mathrm{~cm}, p<0,05)$ e flexibilidade de ombro $(+1,2 \pm 2,1 \mathrm{~cm}, p<0,05)$. Não ocorreram alterações nos fatores de risco cardiovascular, com exceção da redução da pressão arterial diastólica $(-0,9 \pm 6,0 \mathrm{mmHg}$, $p<0,05)$. Entretanto, nos subgrupos com fatores alterados, observou-se reduções significantes das pressões arteriais sistólica e diastólica $(-13,3 \pm 16,9$ e $-5,8 \pm 8,3 \mathrm{mmHg}, \mathrm{p}<0,05$, respectivamente) nos hipertensos, da colesterolemia total $(-19,5 \pm 33,5 \mathrm{mg} / \mathrm{dl}, \mathrm{p}<0,05)$ nos hipercolesterolêmicos e da circunferência da cintura $(-1,0$ $\pm 4,7 \mathrm{~cm}, p<0,05)$ e do índice cintura-quadril $(-0,01 \pm 0,04, p<0,05)$ nos com obesidade central. Conclusão: Numa situação real de atuação, a prescrição de caminhada sem supervisão da prática foi efetiva em melhorar a aptidão fisica da amostra geral e em diminuir o risco cardiovascular específico dos indivíduos com fatores de risco.

Palavras-chave: Treinamento não supervisionado; Fatores de risco; Capacidade física.

\section{Introdução}

O avanço tecnológico, industrial e a praticidade do mundo moderno propiciam que o homem contemporâneo adote, cada vez mais, um estilo de vida sedentário ${ }^{1}$. No Brasil, o inquérito populacional (VIGITEL) conduzido por telefone pelo Ministério da Saúde demonstrou que $14 \%$ da população adulta pratica menos de $10 \mathrm{~min}$ por dia de atividade física de qualquer tipo (lazer, ocupacional ou de locomoção) $)^{2}$. A redução da prática física, no entan- to, tem sido atribuída, principalmente, à redução acentuada das atividades físicas de lazer ${ }^{3}$. Neste sentido, no VIGITEL, apenas 30,3\% dos adultos brasileiros relataram atingir os níveis de atividade física recomendados em atividades de lazer (150 $\mathrm{min} / \mathrm{semana}$ de atividade física leve a moderada ou $75 \mathrm{~min} / \mathrm{semana}$ de atividade vigorosa) $)^{2}$.

A adoção do comportamento sedentário, além de influenciar negativamente os componentes da 
aptidão física, reduzindo a flexibilidade, a capacidade aeróbica, a força e a resistência muscular ${ }^{4-5}$, está diretamente associada à maior prevalência de doenças crônico-degenerativas e dos fatores de risco que as determinam $^{5}$. Por este motivo, a prática de atividade física tem sido recomendada para prevenir e tratar estes fatores, ajudando na redução da obesidade ${ }^{6}$, da pressão arterial $(\mathrm{PA})^{7}$, do colesterol total $(\mathrm{CT})^{8}$ e da glicemia ${ }^{9}$.

Para que estes benefícios sejam conseguidos é necessário que a população se torne ativa. Assim, discute-se como proporcionar esta prática à grande massa populacional sem, no entanto, onerar em demasiado o Estado e a população praticante, e sem submeter esta população a um eventual risco advindo da prática. Nesse sentido, os exercícios físicos não supervisionados aparecem como uma importante estratégia ${ }^{10}$. A caminhada, em especial,

\section{Método}

Foram incluídos neste estudo indivíduos adultos e idosos participantes do Projeto "Exercício e Coração", que é realizado no Parque Fernando Costa, no município de São Paulo. Este projeto tem por objetivo estimular e dar subsídios para a prática segura de atividades físicas visando a melhora e manutenção da saúde e da qualidade de vida da população através da orientação de atividade física não supervisionada.

Os indivíduos envolvidos neste estudo permitiram a utilização de seus dados ao assinarem um termo de consentimento livre e esclarecido aprovado pelo $\mathrm{Co}$ mitê de Ética da Escola de Educação Física e Esporte da Universidade de São Paulo (processo no 2002/10).

Entre os anos de 2001 e 2010, 1030 voluntários foram avaliados no projeto. Destes, 204 retornaram para uma reavaliação e, dentre eles, 186 compareceram para esta reavaliação no prazo de dois e 18 meses da avaliação inicial (o tempo médio entre a avaliação e a reavaliação foi de 7,5 meses). Estes indivíduos compuseram a amostra total do estudo (FIGURA 1).

Para as avaliaçôes, os indivíduos foram orientados a comparecer em jejum de $12 \mathrm{~h}$ e sem praticar exercícios físicos nas $24 \mathrm{~h}$ anteriores. Eles responderam destaca-se como opção devido a sua praticidade, fácil execução, baixo custo e baixo risco para a saúde 5 .

Embora os benefícios da caminhada supervisionada sobre a melhora da aptidão física e o controle dos fatores de risco cardiovascular (FRC) já estejam bem demonstrados ${ }^{11}$ e os benefícios da caminhada não supervisionada também tenham sido observados em estudos especificamente desenhados para este $\mathrm{fim}^{12}$, é importante verificar se estes efeitos são efetivamente verificados numa situação real de intervenção, ou seja, num projeto populacional de incentivo à prática de atividade física, no qual as variáveis intervenientes não são controladas. Desta forma, o objetivo do presente estudo foi avaliar a efetividade da prescrição individualizada de caminhada, sem a supervisão da execução, sobre os FRC e a aptidão física de frequentadores de um parque público.

uma entrevista sobre seus dados pessoais e de saúde, e fizeram medidas antropométricas, metabólicas e de pressão arterial, além de testes de aptidão física. Ao término da avaliação, receberam uma prescrição para caminhar pelo menos três dias na semana, por pelo menos $30 \mathrm{~min}$, numa intensidade leve a moderada, que poderia ser monitorada mantendo-se a frequência cardíaca entre 50 e $80 \%$ da frequência cardíaca de reserva ou caminhando o mais rápido possível desde que conseguissem falar frases longas sem interrupção para respirar. Além disso, os indivíduos foram orientados a fazer exercícios de alongamentos antes e após as caminhadas, o que poderia ser feito em aulas de 20 min oferecidas a todos ou pelo voluntário sozinho sem supervisão. Para isso, o voluntário recebia um folheto com exercícios básicos de alongamento. As três primeiras sessões de caminhada foram monitoradas para orientar a execução correta da prescrição, principalmente o controle da intensidade. Após um período de dois a quatro meses, eles foram convidados a retornar para uma reavaliação. Porém, por se tratar de uma situação real de atuação populacional, muitos não retornaram ou demoraram mais tempo para retornar, como exposto anteriormente. 


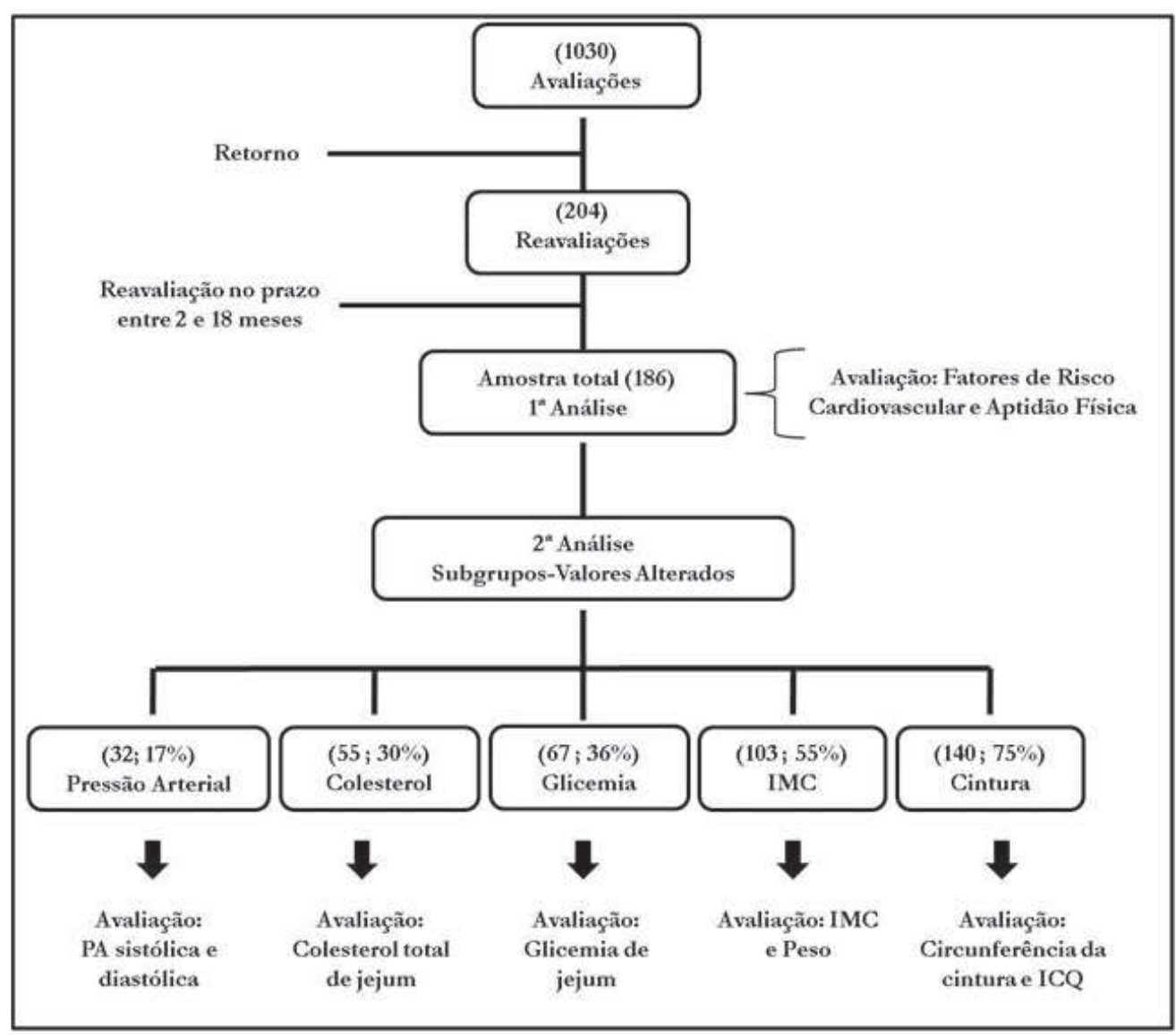

( ) = número de sujeitos; IMC = índice de massa corporal; $I C Q=$ índice cinturaquadril.

FIGURA 1 - Fluxograma de seleção da amostra e procedimentos experimentais.

\section{Medidas}

A entrevista foi composta por questóes sobre os dados pessoais (sexo, idade), dados clínicos (presença de sintomas, doenças cardiovasculares ou outras doenças, hereditariedade, tabagismo e uso regular de medicamentos) e histórico de atividade física praticada (se praticava alguma atividade, qual, quantas vezes por semana, por quanto tempo e em que intensidade).

As medidas antropométricas constaram da medida de peso (balança portátil, Welmy), estatura (estadiômetro simples) e circunferência da cintura (medida com uma fita métrica na altura da cicatriz umbilical) e do quadril (medida na região de maior diâmetro). O índice de massa corporal (IMC) foi calculado pelo quociente entre o peso e o quadrado da estatura e, o índice cintura-quadril (ICQ) pelo do quociente entre as circunferências da cintura e do quadril ${ }^{13}$.

As medidas metabólicas constaram de coleta de sangue para a avaliação do CT e da glicemia de jejum através de amostras sanguíneas obtidas por punção digital e analisadas por monitores automáticos de colesterol (Accutrend ${ }^{\circledR}$ GC - Roche) e de glicose (Advantage II, Roche), respectivamente ${ }^{14-15}$.
A medida da PA foi realizada pelo método auscultatório, empregando-se um esfigmomanômetro aneroide devidamente calibrado. A medida foi realizada três vezes após 5 min de repouso sentado, seguindo-se as recomendações das Diretrizes Nacionais de Hipertensão ${ }^{16}$.

A aptidão física foi avaliada por testes de aptidão aeróbica, muscular e de flexibilidade. Os indivíduos que relataram ter sintomas sugestivos de doenças cardiovasculares, os cardiopatas e aqueles com valores de PA sistólica/diastólica $\geq 160 / 105 \mathrm{mmHg}$ não realizaram os testes de aptidão aeróbica e muscular.

A aptidão aeróbica foi avaliada no teste de marcha estacionária. Nele, os voluntários realizaram o maior número de passos sem deslocamento num período de $2 \mathrm{~min}$, elevando alternadamente os joelhos na altura do ponto médio entre a crista ilíaca e a patela ${ }^{17}$.

A aptidão muscular foi avaliada nos membros superiores e inferiores. Para avaliar a força de membros superiores, os indivíduos realizaram o teste de flexão de cotovelo. Para tanto, eles permaneceram sentados em uma cadeira confortável e realizaram, com o braço dominante, o maior número de repetições possíveis do movimento de flexão e extensão de cotovelo num período de 30 segundos. 
Durante o exercício, as mulheres seguraram um peso de $2 \mathrm{~kg}$ e os homens de $4 \mathrm{~kg}^{17}$. A força de membros inferiores foi avaliada através do teste de impulsão vertical, no qual os voluntários realizaram três saltos verticais sem o auxílio dos membros superiores, que permaneceram estendidos e imóveis acima da cabeça. Foi anotada a maior altura saltada ${ }^{18}$.

Os testes de flexibilidade avaliaram a flexibilidade de ombros e lombar. Para avaliar a flexibilidade de ombros os voluntários posicionaram a mão de preferência atrás do ombro correspondente com a palma da mão direcionada para as costas, buscandose atingir o ponto mais baixo. Ao mesmo tempo, apoiaram o dorso da mão oposta nas costas por baixo da axila, tentando juntar as duas mãos nas costas. Foi considerada a distância existente entre as pontas dos dedos médios alinhados, que recebeu sinal negativo quando as mãos não se encontraram e positivo quando houve sobreposição dos dedos ${ }^{17}$. A flexibilidade lombar foi avaliada pelo teste de sentar e alcançar de Wells e Dillon. Os indivíduos sentaram com os joelhos e braços estendidos e flexionaram o tronco à frente, deslizando as mãos sobre uma fita métrica até o ponto mais distante. Foi considerado o maior valor alcançado em três tentativas ${ }^{19}$.

\section{Análise dos dados}

Os dados foram analisados em duas etapas (FIGURA 1):

$1^{\mathrm{a}}$ análise - Nesta análise, foi avaliado o efeito da caminhada não supervisionada sobre as variáveis hemodinâmicas, metabólicas, antropométricas e de aptidão física da amostra total $(\mathrm{n}=186)$.

\section{Resultados}

A amostra total apresentou idade média de $62 \pm$ 10 anos, variando de 33 a 85 anos. As características da amostra estão apresentadas na TABELA 1. Observa-se um alto risco cardiovascular na população avaliada devido à alta prevalência de doenças e fatores de risco cardiovasculares relatados pelos voluntários.

Os efeitos da caminhada não supervisionada sobre os FRC e a aptidão física na amostra total $\left(1^{\text {a }}\right.$ análise) estão apresentados na TABELA 2. Com relação aos FRC, apenas a PA diastólica diminuiu significantemente entre a avaliação e a reavaliação. Por outro lado, todas as variáveis relacionadas à aptidão física, com exceção da força de membros superiores, melhoraram significantemente na reavaliação. $2^{a}$ análise - Nesta análise, foi avaliado o efeito da caminhada não supervisionada em subgrupos de indivíduos que apresentavam determinado FRC alterado na avaliação. A análise do efeito da caminhada, em cada subgrupo, abrangeu, exclusivamente, o fator que estava alterado. Os subgrupos avaliados foram:

a) Indivíduos com PA alterada - PA sistólica e/ou diastólica > 130/85 mmHg ${ }^{16}-\mathrm{n}=132$;

b) Indivíduos com CT alterado - CT de jejum $>200 \mathrm{mg} / \mathrm{dl}^{15}-\mathrm{n}=55$;

c) Indivíduos com glicemia alterada - glicemia de jejum $>100 \mathrm{mg} / \mathrm{dl}^{14}-\mathrm{n}=67$;

d) Indivíduos com excesso de peso - IMC $>25$ $\mathrm{kg} / \mathrm{m}^{213}-\mathrm{n}=103$;

e) Indivíduos com obesidade central - circunferência da cintura $>94$ e $80 \mathrm{~cm}$ para homens e mulheres, respectivamente ${ }^{13}-\mathrm{n}=140$.

\section{Análise estatística}

A distribuição dos dados foi testada pelos testes de Shapiro-Wilk e Kolmogorov-Smirnov, quando a amostra foi inferior ou superior a 50 indivíduos, respectivamente.

Para as análises inferenciais, os valores absolutos obtidos na avaliação e na reavaliação foram comparados pelo teste t pareado, quando a variável apresentou distribuição normal, ou pelo teste de Wilcoxon, quando sua distribuição não foi normal. Adotou-se o nível de significância de $\mathrm{p}<0,05$.

Todas as análises foram realizadas no pacote estatístico SPSS for Windows (Statistical Package for Social Sciences, version 13.0, Chicago, IL, USA). Os resultados estão apresentados como média \pm DP.

Na TABELA 3 estão apresentados os resultados da $2^{\mathrm{a}}$ análise. No subgrupo com PA alterada, tanto a PA sistólica quanto diastólica diminuíram significantemente. No subgrupo com colesterolemia alterada, a concentração de colesterol total diminuiu significantemente. No subgrupo com glicemia alterada, nenhuma modificação significante foi observada. No subgrupo com excesso de peso, a massa corporal e o IMC tenderam a diminuir ( $\mathrm{p}$ $=0,051$ e 0,067 , respectivamente) e, no grupo com obesidade central, tanto a circunferência da cintura quanto o índice cintura quadril reduziram significantemente. 
TABELA 1 - Características gerais da amostra na avaliação.

\begin{tabular}{lc}
\hline Perfil da Amostra $(\mathbf{N}=\mathbf{1 8 6})$ & $\mathbf{N}(\%)$ \\
\hline Sexo & $46(24,7 \%)$ \\
$\quad$ Homens & $140(75,3 \%)$ \\
$\quad$ Mulheres & \\
Faixa Etária & $6(3,2 \%)$ \\
30 a 40 anos & $73(39,5 \%)$ \\
40 a 60 anos & $106(57,3 \%)$ \\
Mais de 60 anos & \\
Doenças Conhecidas & $28(15,1 \%)$ \\
Cardiopatia relatada & \\
Fatores de Risco Conhecidos & $150(80,6 \%)$ \\
Sexo/Idade (homens > 45 e mulheres > 55 anos) & $68(36,6 \%)$ \\
Hereditariedade (pai, máe ou irmáos) & $10(5,4 \%)$ \\
Tabagismo Atual ou nos último 6 meses & $65(34,9 \%)$ \\
Hipertensáo (diagnóstico médico ou medicamento) & $69(37,1 \%)$ \\
Dislipidemia (diagnóstico médico ou medicamento) & $20(10,8 \%)$ \\
Diabetes (diagnóstico médico ou medicamento) &
\end{tabular}

TABELA 2 - Efeito da caminhada não supervisionada acompanhada de exercícios de alongamento sobre os fatores de risco cardiovasculares e a aptidão física de usuários de um parque público.

\begin{tabular}{|c|c|c|c|c|c|}
\hline Variáveis & $\mathbf{N}$ & Avaliação & Reavaliação & $\mathbf{p}$ & \multirow{10}{*}{ 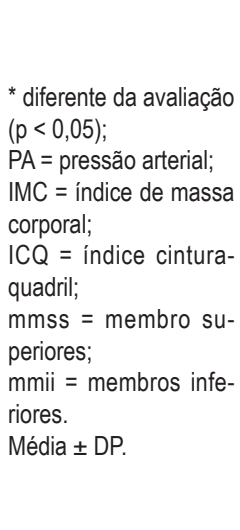 } \\
\hline \multicolumn{5}{|l|}{ Fatores de Risco Cardiovascular } & \\
\hline PA sistólica (mmHg) & 154 & $126 \pm 16$ & $124 \pm 1,6$ & 0,187 & \\
\hline PA diastólica (mmHg) & 159 & $80 \pm 9$ & $79 \pm 8^{*}$ & 0,017 & \\
\hline Colesterolemia (mg/dl) & 129 & $197 \pm 31$ & $195 \pm 30$ & 0,428 & \\
\hline Glicemia (mg/dl) & 157 & $100 \pm 16$ & $102 \pm 17$ & 0,052 & \\
\hline Peso $(\mathrm{kg})$ & 153 & $66,9 \pm 11,6$ & $66,9 \pm 11,4$ & 0,906 & \\
\hline $\operatorname{IMC}\left(\mathrm{kg} / \mathrm{m}^{2}\right)$ & 160 & $26,6 \pm 3,8$ & $26,6 \pm 3,8$ & 0,297 & \\
\hline Cintura $(\mathrm{cm})$ & 162 & $93,7 \pm 11,4$ & $93,3 \pm 10,9$ & 0,330 & \\
\hline ICQ & 161 & $0,92 \pm 0,07$ & $0,91 \pm 0,07$ & 0,492 & \\
\hline \multicolumn{6}{|l|}{ Aptidão Física } \\
\hline Marcha (passos) & 153 & $100 \pm 21$ & $108 \pm 12^{*}$ & 0,000 & \\
\hline Flexibilidade lombar $(\mathrm{cm})$ & 161 & $23,6 \pm 9,3$ & $24,7 \pm 9,6 *$ & 0,002 & \\
\hline Flexibilidade de ombro $(\mathrm{cm})$ & 152 & $-1,12 \pm 7,39$ & $0,11 \pm 7,57^{*}$ & 0,000 & \\
\hline Resistência de mmss (rep) & 150 & $19,8 \pm 4,8$ & $20,1 \pm 4,5$ & 0,275 & \\
\hline Força de mmii $(\mathrm{cm})$ & 140 & $17,6 \pm 5,8$ & $18,1 \pm 6,0 *$ & 0,028 & \\
\hline
\end{tabular}


TABELA 3 - Efeito da caminhada não supervisionada sobre os fatores de risco cardiovascular alterados em subgrupos de indivíduos com fatores específicos modificados.

* diferente da avaliação

$(p<0,05)$;

$\mathrm{PA}=$ pressão arterial;

IMC = índice de massa corporal;

$\mathrm{ICQ}=$ índice cinturaquadril.

Média \pm DP.

\begin{tabular}{llcccc}
\hline Fator Alterado & Variáveis & N & Avaliação & Reavaliação & p \\
\hline \multirow{2}{*}{ PA } & PA sistólica $(\mathrm{mmHg})$ & 32 & $151 \pm 19$ & $137 \pm 16^{*}$ & 0,000 \\
& PA diastólica $(\mathrm{mmHg})$ & 32 & $94 \pm 7$ & $88 \pm 7^{*}$ & 0,000 \\
Colesterol & Colesterolemia $(\mathrm{mg} / \mathrm{dl})$ & 55 & $228 \pm 25$ & $209 \pm 34^{*}$ & 0,000 \\
Glicemia & Glicemia $(\mathrm{mg} / \mathrm{dl})$ & 67 & $113 \pm 16$ & $114 \pm 19$ & 0,951 \\
& Peso $(\mathrm{kg})$ & 103 & $74,8 \pm 10,3$ & $73,3 \pm 10,1$ & 0,051 \\
Excesso de peso & IMC $\left(\mathrm{kg} / \mathrm{m}^{2}\right)$ & 103 & $28,9 \pm 3,0$ & $28,7 \pm 3,1$ & 0,067 \\
& Cintura $(\mathrm{cm})$ & 140 & $96,6 \pm 10,0$ & $95,6 \pm 10,3^{*}$ & 0,011 \\
Cesidade & ICQ & 139 & $0,93 \pm 0,06$ & $0,92 \pm 0,07^{*}$ & 0,043 \\
\hline
\end{tabular}

\section{Discussão}

Os principais resultados deste estudo foram que a prescrição individualizada de caminhada sem supervisão da prática foi efetiva em: a) melhorar a aptidão física da amostra geral; b) proporcionar benefícios específicos sobre os FRC em indivíduos que apresentavam fatores alterados.

\section{Efeito da caminhada não supervisionada sobre a aptidão física}

No presente estudo, a intervenção proposta aumentou a capacidade aeróbica, a flexibilidade lombar e de ombros e a força muscular de membros inferiores dos usuários do parque. Estes resultados demonstram que, mesmo em condiçõos reais de intervenção e sem um controle de variáveis intervenientes, a prescrição da prática de caminhada realizada sem supervisão é efetiva para melhorar a aptidão física de uma população que frequenta locais públicos, como um parque.

O aumento da aptidão aeróbica com o treinamento aeróbico supervisionado é a adaptação clássica ${ }^{4}$. Estudos especificamente desenhados para verificar o efeito de programas não supervisionados de treinamento aeróbico também têm consistentemente demonstrado benefícios sobre a aptidão aeróbica. KING et al. ${ }^{20}$ observaram aumento em torno de $5 \%$ nesta aptidão com o treinamento não supervisionado. Este resultado é um pouco menor que o encontrado no presente estudo $(8,1 \%)$. Cabe destacar, entretanto, que os testes aplicados para avaliar esta capacidade foram diferentes entre os estudos, o que pode limitar a comparação dos resultados.
Quanto à flexibilidade, houve melhora de 5\% na flexibilidade lombar e de $1,23 \mathrm{~cm}$ na flexibilidade de ombros. Estes valores são um pouco inferiores aos observados em outros estudos empregando programas não supervisionados de exercícios ${ }^{21-22}$. FARINATTI et al. ${ }^{21}$ observaram aumento de $13 \%$ na flexibilidade lombar após um período de treinamento não supervisionado em casa. De forma semelhante, PANISI et al..$^{22}$ observaram aumento de $1,66 \mathrm{~cm}$ na flexibilidade de ombros. Os menores valores observados no presente estudo podem se dever ao fato da prescrição dada aos voluntários enfatizar a caminhada e não especificamente os exercícios de alongamento, apesar destes exercícios serem estimulados e, inclusive, poderiam ser feitos em aulas. É interessante observar, no entanto, que mesmo sem uma prescrição específica para o ganho de flexibilidade, esta melhora ocorreu, o que sugere que um incentivo já pode trazer benefícios em condições reais de atuação populacional.

Embora a prescrição tenha incluído apenas caminhada e alongamento, foi possível observar melhora da força de membros inferiores. Existem evidências que a prática de caminhada pode aumentar a força de membros inferiores em indivíduos idosos ${ }^{23}$, embora ganhos maiores sejam obtidos com o treinamento resistido ${ }^{24}$.

Cabe ressaltar que não houve aumento da resistência de membros superiores, o que já era esperado, visto que a prescrição de caminhada e alongamentos não visava a melhora desta capacidade física. De fato, a variável resistência de membros superiores pode ser considerada uma variável controle neste estudo e, neste sentido, a ausência de efeitos sobre 
ela fortalece o pressuposto de que os efeitos positivos encontrados nas outras capacidades físicas decorreram da intervenção realizada e não de outros fatores, como a adaptação aos testes ou o passar do tempo, entre outros.

\section{Efeito da caminhada não supervisionada sobre os fatores de risco cardiovascular}

$\mathrm{Na}$ amostra total, a caminhada não supervisionada não promoveu efeitos sobre os FRC, exceto a PA diastólica que diminuiu após o período de intervenção. A ausência de efeito sobre estes fatores pode estar associada aos baixos níveis iniciais destes fatores quando considerada a amostra como um todo. É sabido que indivíduos que possuem FRC em níveis normais apresentam alterações menos expressivas destes fatores em resposta à prática de exercícios físicos ${ }^{8,25}$.

Considerando-se a PA, apenas $17 \%$ da amostra apresentavam valores de PA alterados na avaliação. De fato, diversos estudos sobre os efeitos do treinamento aeróbico supervisionado têm demonstrado que os efeitos hipotensores deste treinamento são maiores em indivíduos hipertensos ou com valores mais altos de $\mathrm{PA}^{26}$. Este mesmo comportamento pode ser esperado após o treinamento não supervisionado ${ }^{12}$ e, neste sentido, a redução significante, tanto da PA sistólica quanto diastólica, no subgrupo que apresentava valores de PA elevados na avaliação corrobora com este conhecimento. De fato, neste subgrupo, o efeito hipotensor teve magnitude de 14 e $6 \mathrm{mmHg}$ para PA sistólica e diastólica, respectivamente, o que é superior ao esperado após o treinamento supervisionado em hipertensos ${ }^{26}$. Além disso, esta redução reflete uma redução significante no risco de desenvolvimento de doença da artéria coronária e acidente vascular cerebral ${ }^{25}$.

Considerando-se a colesterolemia, ela também não se alterou na amostra total, mas diminuiu significantemente no subgrupo com CT elevado. Novamente, apenas $30 \%$ da amostra total apresentavam valores de colesterol elevados, o que pode explicar a ausência de efeito devido aos baixos níveis iniciais, visto que o efeito do treinamento sobre o colesterol também é maior em sujeitos com níveis alterado ${ }^{27}$. A redução encontrada no subgrupo com CT elevado foi de $8 \%$, o que pode refletir uma importante cardioproteção, visto que a diminuição de $11 \%$ do CT diminui em $49 \%$ a chance de eventos coronarianos em cinco anos ${ }^{28}$.
O programa de exercícios proposto não influenciou os níveis de glicemia nem na amostra total, nem nos indivíduos que possuíam esses níveis elevados. Este resultado pode parecer estranho a princípio, visto que é bastante aceito que programas de exercício aeróbico melhoram a sensibilidade à insulina e a tolerância à glicose, diminuindo a glicemia sanguínea em indivíduos com glicemia alterada? Porém, outros estudos sem supervisão da atividade também não observaram redução significante da glicemia $^{22,29}$. Uma possível explicação para a ausência de efeito pode estar relacionada à forte influência da dieta na glicemia ${ }^{30} \mathrm{o}$ que não foi controlado no presente estudo. Estes resultados, portanto, sugerem que os programas de intervenção devam incluir uma atuação nutricional, principalmente se englobarem indivíduos com glicemia elevada.

Considerando o excesso de peso e a obesidade central, diferentemente dos outros FRC, a prevalência de alteração foi grande na população total (75\%). Apesar disso, os efeitos da intervenção não foram observados na amostra total. No entanto, houve uma forte tendência à redução da massa corporal e do IMC nos indivíduos com excesso de peso e houve diminuição significante da circunferência da cintura e do índice cintura-quadril nos sujeitos com obesidade central. Há evidências na literatura que a atividade física realizada com intensidade moderada e volume entre 150 e 250 minutos por semana seja efetiva para a manutenção ou redução do peso corporal ${ }^{6}$. No presente estudo, o volume de treinamento foi suficiente para manter a massa corporal e diminuir a obesidade central. Possivelmente, a ausência de controle ou orientação dietética tenha impedido um efeito mais expressivo sobre o IMC, visto que o controle da dieta alimentar parece ser imprescindível em programas que almejam a redução do peso corporal ${ }^{13}$. É interessante, no entanto, apontar que houve redução dos índices de obesidade central da mesma forma que em outros estudos não supervisionados ${ }^{12,21}$. Esta redução é de suma importância, uma vez que o acúmulo de gordura nesta região está diretamente associado à ocorrência de disfunções metabólicas ${ }^{13}$.

Analisando-se em conjunto todos os efeitos da intervenção proposta sobre os FRC é possível sugerir que a prescrição de caminhada quando realizada sem supervisão numa situação real de intervenção populacional e, portanto, sem controle de fatores intervenientes foi efetiva em reduzir o risco cardiovascular dos indivíduos que possuíam este risco aumentado, através da atuação na redução específica do fatores de risco presente. 


\section{Limitações}

$\mathrm{Na}$ ciência, os conhecimentos precisam ser testados translacionalmente ${ }^{31}$. Assim, conhecimentos obtidos na pesquisa básica, precisam ser testados em pesquisas aplicadas e, posteriormente, precisam ser testados em situações reais de prática. Obviamente, em cada um destes níveis, as pesquisas apresentam graus de controle e limitaçôes diferentes. $\mathrm{O}$ estudo exposto neste artigo refere-se ao terceiro tipo, ou seja, à testagem de um conhecimento, o efeito da caminhada sobre os FRC e aptidão física, numa situação real de prática em nível populacional. Este tipo de estudo apresenta diversas limitações metodológicas, impostas pela situação real, que impede o controle de variáveis intervenientes, mas, por outro lado, aumenta expressivamente a validade ecológica dos achados. Assim, os resultados precisam ser interpretados à luz deste tipo de estudo.

Neste sentido, no presente estudo, as coletas duraram vários anos, de modo que diferentes avaliadores participaram da coleta em momentos diferentes não sendo possível avaliar a reprodutibilidade de dados entre eles. No entanto, todos passaram por treinamento prévio seguido de avaliação de sua performance para garantir a padronização das medidas e reduzir a variação interobservador. Por se tratar de uma atuação real, não foi possível, por questôes éticas, a realização de um grupo controle sem exercício tendo em vista os benefícios esperados desta prática. Da mesma forma, não foi possível controlar variáveis como dieta, medicamentos ou mesmo outras atividades que os sujeitos quisessem realizar. No entanto, estes aspectos caracterizam a validade ecológica do estudo, ou seja, a intervenção obteve seus efeitos mesmo sem controlar as variáveis intervenientes. Em relação aos aspectos metodológicos, por ser um estudo populacional feito num parque público, os testes foram escolhidos em função das condiçôes do local e da população. Outros testes poderiam ter trazido informaçóes adicionais ou mais precisas, mas não puderam ser realizados. Como era um projeto de participação voluntária, os participantes nem sempre cumpriam todas as etapas do projeto, o que resultou em grande diferença de número de sujeitos entre as avaliaçōes e as reavaliações e no tempo entre a avaliação e a reavaliação. Por ser um trabalho não supervisionado, a prática da caminhada foi controlada pelos próprios voluntários, de modo que não se pode garantir se a prescrição foi realmente seguida (frequência, duração e intensidade), embora todos os voluntários relataram tê-la seguido, pelo menos parcialmente.

Com base nos resultados pode-se concluir que a prescrição de caminhada sem supervisão da prática, quando realizada numa situação real de intervenção populacional, foi efetiva em melhorar a aptidão física da amostra geral e em reduzir o risco cardiovascular específico de sujeitos com fatores de risco alterados. Estes resultados demonstram a eficácia deste tipo de atuação na melhora da aptidão física e redução do risco cardiovascular da população, sugerindo que programas deste tipo devam ser estimulados em locais públicos de prática física.

\begin{abstract}
Unsupervised walking prescription, cardiovascular risk and physical fitness

Objective: To evaluate, at a real practical condition, the effects of individualized prescription of walking without supervision of practice on cardiovascular risk and fitness in users of a public park. Methods: One hundred, eighty six subjects (62 \pm 10 years) were instructed to walk at least 3 times/week, during $30 \mathrm{~min}$, at an intensity of $50-80 \%$ of heart rate reserve and encouraged to realize stretching exercises before and after walking. Physical fitness and cardiovascular risk factors were evaluated pre and postintervention. Data analyze was divided in 2 phases: 1) role sample analysis; and 2) analysis on subjects with altered cardiovascular risk factors. Data were compared by paired t test. Results: Considering the whole sample, physical fitness improved in the following tests: stationary gate $(8.1 \pm 14.5$ paces, $p<$ $0.05)$, vertical jump $(0.5 \pm 2.7 \mathrm{~cm}, p<0.05)$, lumbar flexibility $(1.1 \pm 4.7 \mathrm{~cm}, p<0.05)$ and shoulder flexibility $(1.2 \pm 2.1 \mathrm{~cm}, \mathrm{p}<0.05)$. No significant change was observed in cardiovascular risk factors, excepted by a reduction on diastolic blood pressure $(-0.9 \pm 6.0 \mathrm{mmHg}, \mathrm{p}<0.05)$. On the other hand, considering the subjects with altered cardiovascular risk factors, a significant reduction was observed on systolic and diastolic blood pressures $(-13.3 \pm 16.9$ and $-5.8 \pm 8.3 \mathrm{mmHg}, \mathrm{p}<0.05$, respectively)
\end{abstract}


in hypertensive subjects, on total cholesterol $(-19.5 \pm 33.5 \mathrm{mg} / \mathrm{dl}, \mathrm{p}<0.05)$ in hypercholesterolemic subjects, and on waist circumference $(-1.0 \pm 4.7 \mathrm{~cm}, p<0.05)$ and waist-hip index $(0.01 \pm 0.04, p<$ $0.05)$ in subjects with central obesity. Conclusion: Under real practical circumstances, the prescription of unsupervised walking was effective in improving physical fitness in general sample and in reducing the specific cardiovascular risk in subjects who have altered cardiovascular risk factors.

KEY WORDS: Unsupervised training; Risk factors; Physical capacity.

\section{Referências}

1. Florindo AA, Guimaraes VV, Cesar CL, et al. Epidemiology of leisure, transportation, occupational, and household physical activity: prevalence and associated factors. J Phys Act Health. 2009;6:625-32.

2. Brasil. Ministério da Saúde. VIGITEL Brasil 2011: vigilância de fatores de risco para doenças crônicas por inquérito telefônico. Brasília: Ministério da Saúde; 2012.

3. Gomes VB, Siqueira KS, Sichieri R. Atividade física em uma amostra probabilistica da população do Município do Rio de Janeiro. Cad Saude Publica. 2001;17:969-76.

4. Baker MK, Atlantis E, Fiatarone Singh MA. Multi-modal exercise programs for older adults. Age and ageing. 2007;36:375-81.

5. Leon A. Physical activity and cardiovascular health: a national consensus. Champaingn: Human Kinetcs; 1997.

6. Donnelly JE, Blair SN, Jakicic JM, Manore MM, Rankin JW, Smith BK. American College of Sports Medicine Position Stand. Appropriate physical activity intervention strategies for weight loss and prevention of weight regain for adults. Med Sci Sports Exerc. 2009;41:459-71.

7. Alves LL, Forjaz CL. Influência da intensidade e do volume do treinamento aeróbico na redução da pressão arterial de hipertensos. Rev Bras Ciênc Mov. 2007;15:115-22.

8. Lamarche B, Despres JP, Pouliot MC, et al. Is body fat loss a determinant factor in the improvement of carbohydrate and lipid metabolism following aerobic exercise training in obese women? Metabolism. 1992;41:1249-56.

9. Sigal RJ, Kenny GP, Wasserman DH, Castaneda-Sceppa C, White RD. Physical activity/exercise and type 2 diabetes: a consensus statement from the American Diabetes Association. Diabetes Care. 2006;29:1433-8.

10. Carlson JJ, Johnson JA, Franklin BA, VanderLaan RL. Program participation, exercise adherence, cardiovascular outcomes, and program cost of traditional versus modified cardiac rehabilitation. Am J Cardiol. 2000;86:17-23.

11. Cornelissen VA, Fagard RH. Effects of endurance training on blood pressure, blood pressure-regulating mechanisms, and cardiovascular risk factors. Hypertension. 2005;46:667-75.

12. Nunes AP, Rios AC, Cunha GA, Barretto AC, Negrao CE. Efeitos de um programa de exercício físico não-supervisionado e acompanhado a distância, via internet, sobre a pressão arterial e composição corporal em indivíduos normotensos e pré-hipertensos. Arq Bras Cardiol. 2006;86:289-96.

13. Associação Brasileira para o Estudo da Obesidade e da Síndrome Metabólica. Diretrizes Brasileiras de Obesidade. $3 a$ ed. Itapevi: AC Farmacêutica; 2009.

14. American Diabetes Association. Diagnosis and classification of diabetes mellitus. Diabetes Care. 2012;35 Suppl 1:S64-S71.

15. Sposito AC, Caramelli B, Fonseca FA, et al. IV Diretriz Brasileira sobre Dislipidemias e Prevenção da Aterosclerose: Departamento de Aterosclerose da Sociedade Brasileira de Cardiologia. Arq Bras Cardiol. 2007;88 Suppl 1:2-19.

16. Sociedade Brasileira de Hipertensão. VI Diretrizes Brasileiras de Hipertensão Arterial. Rev Hipertensão. 2010;13.

17. Rikli R, Jones C. Development and validation of a functional fitness test for community-residing older adults. J Aging Phys Act. 1999;7:129-61.

18. Soares J. Medidas de força muscular. In: Matsudo V, organizador. Testes em ciências do esporte. 5a ed. São Caetano do Sul: CELAFISCS; 1995. p. 73-7.

19. Canadian Society for Exercise Physiology. The Canadian Physical Activity, Fitness \& Lifestyle Approach: CSEP Health-Related Appraisal \& Counseling Strategy. 3rd ed. Ottawa: Health Canada; 2003.

20. King AC, Haskell WL, Taylor CB, Kraemer HC, DeBusk RF. Group- vs home-based exercise training in healthy older men and women: a community-based clinical trial. JAMA. 1991;266:1535-42.

21. Farinatti PT, Oliveira RB, Pinto VL, Monteiro WD, Francischetti E. Programa domiciliar de exercícios: efeitos de curto prazo sobre a aptidão física e pressão arterial de indivíduos hipertensos. Arq Bras Cardiol. 2005;84:473-9. 
Queiroz ACC, et al.

22. Panisi P, Pádua PR, Martins VMS, et al. Efeito da prescrição de caminhada sem supervisão da prática num parque público. Rev Bras Ativ Fis Saúde. 2012;17:423-33.

23. Simons R, Andel R. The effects of resistance training and walking on functional fitness in advanced old age. J Aging Health. 2006;18:91-105.

24. Ferketich AK, Kirby TE, Alway SE. Cardiovascular and muscular adaptations to combined endurance and strength training in elderly women. Acta Physiol Scand. 1998;164:259-67.

25. Chobanian AV, Bakris GL, Black HR, et al. The seventh report of the joint national committee on prevention, detection, evaluation, and treatment of high blood pressure. JAMA. 2003;289:2560-72.

26. Cornelissen VA, Smart NA. Exercise training for blood pressure: a systematic review and meta-analysis. J Am Heart Assoc. 2013. Epub 2013 Mar 26. doi: 10.1161/JAHA.112.004473. Pubmed PMID: 23525435.

27. Tran ZV, Weltman A, Glass GV, Mood DP. The effects of exercise on blood lipids and lipoproteins: a meta-analysis of studies. Med Sci Sports Exerc. 1983;15:393-402.

28. Trial of clofibrate in the treatment of ischaemic heart disease. Five-year study by a group of physicians of the Newcastle upon Tyne region. Br Med J. 1971;4:767-75.

29. Avery MD, Leon AS, Kopher RA. Effects of a partially home-based exercise program for women with gestational diabetes. Obstet Gynecol. 1997;89:10-5.

30. Ross R, Dagnone D, Jones PJ, et al. Reduction in obesity and related comorbid conditions after diet-induced weight loss or exercise-induced weight loss in men. A randomized, controlled trial. Ann Intern Med. 2000;133:92-103.

31. Curry SH. Translational science: past, present, and future. BioTechniques. 2008;44:ii-viii.

\section{Agradecimentos}

Os autores agradecem todos os voluntários que participaram deste estudo, à Administração do Parque Fernando Costa, à Associação de Ambientalistas e Amigos do Parque da Água Branca (ASSAMAPAB), ao Fundo Social do Estado de São Paulo e ao apoio financeiro da Pró-Reitoria de Cultura e Extensão da Universidade de São Paulo (Fundo de Extensão, Bolsa Aprendendo com Cultura e Extensão).

\begin{tabular}{r|r} 
ENDEREÇo & \\
Andreia Cristiane Carrenho Queiroz & \\
Escola de Educação Física e Esporte - USP & Recebido para publicação: 02/05/2013 \\
Av. Prof. Mello Moraes, 65 & Aceito: 28/08/2013 \\
05508-030 - São Paulo - SP - BRASIL & \\
e-mail: andreiaqueiroz@usp.br &
\end{tabular}

386 • Rev Bras Educ Fís Esporte, (São Paulo) 2013 Jul-Set; 27(3):377-86 